\title{
Simulation of Nonlinear Thermomechanical Waves with an Empirical Low Dimensional Model
}

\author{
Linxiang Wang ${ }^{1}$ and Roderick V.N. Melnik ${ }^{2}$ \\ 1 MCI, Faculty of Science and Engineering, \\ University of Southern Denmark, \\ Sonderborg, DK-6400, Denmark \\ 2 Centre for Coupled Dynamics \& Complex Systems, \\ Wilfrid Laurier University, \\ 75 University Avenue West, \\ Waterloo, ON, Canada, N2L 3C5
}

\begin{abstract}
In this paper we analyse the performance of a low dimensional model for the nonlinear thermo-mechanical waves. The model has been obtained by using proper orthogonal decomposition methods combined with a Galerkin projection. First, we analyse the original PDE model in order to obtain the system states at many time instances. Then, by using an empirical orthogonal basis extracted from our numerical results, we construct an empirical low dimensional model. Finally, we compare the results obtained with the original PDE model and those obtained with our low-dimensional model. These comparisons are carried out for mechanically induced phase transformations in a shape-memory alloy rod.
\end{abstract}

Keywords: Nonlinear waves, thermo-mechanical dynamics, proper orthogonal decompositions, Galerkin projections.

\section{Introduction}

The field of smart material systems is rapidly developing. Due to their unique properties, the smart materials have attracted an increasing attention from mathematicians, physicists, control theorists, and engineers. Smart materials such as piezoelectrics, shape memory alloys, and magnetostrictive materials can sense and respond to external stimuli. They can also be used as actuators. Ultimately, one would like to achieve a certain degree of control over phenomena associated with the complex behaviour of these materials ([17,6] and references therein). As a result, there is an increasing number of efforts to construct simple and robust mathematical models describing the dynamics of smart materials and such that would be relatively easy to amend to control.

Control strategies for many dynamic systems described by nonlinear ODEs are well developed, in particular in those cases where the dimension of the con- 
trolled system is not large [2,13]. At the same time, existing mathematical models for the dynamical behaviour of smart materials are typically based on a set of coupled nonlinear PDEs. For example, the mathematical models for the shape memory alloys are formulated as a system of PDEs that couples thermal and elastic fields, while the models for the piezoelectric materials are formulated as a system of PDEs that couples electric and elastic fields [11,19]. All such models are infinite dimensional and one of the ways to deal with such models is to discretize them in space and apply the method of lines to the result. However, due to the coupling between multi-physics fields and system nonlinearities, the number of nodes for the spatial discretization needs to be sufficiently large. This leads to computational difficulties due to the fact that the resultant large system of ODEs is usually stiff. Even in the case of linear PDEs, control issues of the resulting models are highly non-trivial [18, 14, 4, 9, Hence, it seems natural to try to approximate PDE systems such as those arising in the description of smart material systems by a lower dimensional ODE systems. One way to do that is to use the Proper Orthogonal Decomposition (POD). This is a very efficient tool for this purpose as soon as a collection of system states is available. This idea has been applied to many active control problems involving fluid flows (see 11,14, 5, 15] and references therein). In its essence, the POD methodology is analogous to the principal component analysis, techniques based on the singular value decomposition, or the Karhunen-Loeve decomposition [8, 5, 15.

In what follows, we propose a low dimensional model for the nonlinear thermomechanical waves, describing the dynamics of shape memory alloys. The model is constructed on the basis of the numerical results obtained from the original system of coupled nonlinear PDEs. The dynamical behavior of the considered system is simulated by the empirical low dimensional model, and the performance of the low dimensional model is compared to the original PDE model. It is shown that the empirical low dimensional captures all the characteristic features of the material.

\section{The Original PDE Model}

Many smart materials encountered in applications have been extensively investigated by both experimentalists and theoreticians. Today, mathematical models for the 1D shape memory alloys rods (or wires) are well established on the basis of the modified Ginzburg-Landau theory. The well known Falk model has been constructed on the basis of conservation laws for linear momentum and energy, and thermo-dynamical consistency. To model the coupled thermo-mechanical wave interactions and the first order phase transitions in the shape memory alloys, we use the following 1D mathematical model [3, 11, 19]:

$$
\begin{aligned}
\rho \frac{\partial^{2} u}{\partial t^{2}} & =\frac{\partial}{\partial x}\left(k_{1}\left(\theta-\theta_{1}\right) \frac{\partial u}{\partial x}-k_{2}\left(\frac{\partial u}{\partial x}\right)^{3}+k_{3}\left(\frac{\partial u}{\partial x}\right)^{5}\right)+F, \\
c_{v} \frac{\partial \theta}{\partial t} & =k \frac{\partial^{2} \theta}{\partial x^{2}}+k_{1} \theta \frac{\partial u}{\partial x} \frac{\partial v}{\partial x}+G,
\end{aligned}
$$


where $u$ is the displacement, $\theta$ is the temperature, $\rho$ is the density, $k_{1}, k_{2}, k_{3}, c_{v}$ and $k$ are re-normalized material-specific constants, $\theta_{1}$ is the reference temperature for 1D martensitic transformations, and $F$ and $G$ are distributed mechanical and thermal loadings.

It is well known that even in this one-dimensional case, the analysis of the system is far from trivial due to the strong nonlinear coupling between thermal and elastic fields. Thermal and mechanical hysteresis effects and complicated phase transformations are the phenomena that need to be dealt with. Following our previous works [10,11, 19, we re-write our original system in a way convenient for computational implementation:

$$
\begin{gathered}
c_{v} \frac{\partial \theta}{\partial t}=k \frac{\partial^{2} \theta}{\partial x^{2}}+k_{1} \theta \epsilon \frac{\partial v}{\partial x}+G, \quad \frac{\partial \epsilon}{\partial t}=\frac{\partial v}{\partial x} \\
\rho \frac{\partial v}{\partial t}=\frac{\partial}{\partial x}\left(k_{1}\left(\theta-\theta_{1}\right) \epsilon-k_{2} \epsilon^{3}+k_{3} \epsilon^{5}\right)+F,
\end{gathered}
$$

where $\epsilon=\partial u / \partial x, v=\partial u / \partial t$.

\section{The Construction of a Low Dimensional Model}

\subsection{Orthogonal Basis}

The first step in constructing a low dimensional model is to construct an effective basis for the approximation of the system states. This step is equivalent to the one described in [12] where Eq.(2) is rewritten in a general dynamical system form

$$
\frac{\partial \mathcal{U}(x, t)}{\partial t}=\mathcal{F}(\mathcal{U}(x, t)),
$$

and $\mathcal{U}(x, t)$ is the sought-for vector function with components $\epsilon, v$ and $\theta$. This function depends on the spatial position continuously in a given domain $\Omega$ and $\mathcal{F}$ is a nonlinear function of $\mathcal{U}$, and the first, second order derivatives of $\mathcal{U}$. At this stage, loadings are not included in the above model.

The POD is concerned with the possibility to find a set of orthonormal basis functions $\phi_{j}(x), j=1, \ldots, P$ which are optimal in the sense that the $P$ dimensional approximation

$$
\mathcal{U}_{P}(x, t)=\sum_{i=1}^{P} a_{i}(t) \phi_{i}(x)
$$

gives the best approximation to the function $\mathcal{U}(x, t)$ among all those $P$ dimensional approximations, in the least square sense [8, 5, 16]. As usual, here $a_{i}$ are the general Fourier coefficients for $\phi$, that are functions of time. In other words, the idea of POD applied here is based on a choice of the basis functions $\phi$ to maximize the mean projection of the function $\mathcal{U}(x, t)$ on $\phi$

$$
\max _{\phi \in L_{2}(\Omega)} \frac{E\left(|\langle\mathcal{U}, \phi\rangle|^{2}\right)}{\|\phi\|^{2}}
$$


where $E(\cdot)$ denotes the mean value functional, and $\langle\cdot\rangle$ is the inner product [5, 16. Finally, the maximization problem leads to the following eigenvalue problem

$$
\int_{\Omega} E\left(\mathcal{U}(x) \mathcal{U}\left(x^{\prime}\right)\right) \phi\left(x^{\prime}\right) d x^{\prime}=\lambda \phi(x),
$$

whose kernel $\mathcal{K}=E\left(\mathcal{U}(x) \mathcal{U}\left(x^{\prime}\right)\right)$ can be interpreted as the auto-covariance function of the two points $x$ and $x^{\prime}$ (see [5, 16] and references therein).

In practice, the states of the above system could be obtained by either experimental measurements or numerical simulation with suitable initial and boundary conditions, so $\mathcal{U}(x, t)$ will be a discrete function in both time and space. If we assume that the system states are available at $N$ different time instances, we will call the system state at the $i^{\text {th }}$ time instance $U^{i}$ as the $i^{\text {th }}$ snapshot. In the discrete form, each snapshot can be written as a column vector with $M$ entries, where $M$ is the number of nodes for spatial discretization.

In order to construct the orthonormal basis, all the snapshots need to be collected in one matrix $U=\left\{U^{i}, i=1, \ldots, N\right\}$, and we can put each snapshot as one column in the collection matrix so that the collection $U$ will be a $M \times N$ matrix. Then, the orthonormal basis for the given collection $U$ can be calculated by the singular value decomposition as follows

$$
U=L S R^{T}
$$

where $L$ is $M \times M$ orthonormal matrix, $R$ is a $N \times N$ orthonormal matrix, $S$ is a $M \times N$ matrix with all elements zero except along the diagonal and those non-zero elements are arranged in a decreasing order along the diagonal (the singular values of $U$ with associated eigenvectors in $L$ and $R$ ).

If we let $S R^{T}=Q^{T}$ in the singular value decomposition, then $U=L Q^{T}$. Let then $\phi_{k}$ be the $k^{t h}$ column of $L$ and $a_{k}$ be the $k^{\text {th }}$ row of $Q$, so that the matrix $U$ 's singular value decomposition can be rewritten as

$$
U=\sum_{k=1}^{m} a_{k} \phi_{k}
$$

where $m=\min (N, M)$ is the rank of the collection matrix $U$. This approximation is what we are looking for in Eq.(44). Following [7,5], we note that the lower dimensional approximation of the matrix $U$ can be easily obtained by just keeping the first few largest singular values and their associated eigenvectors in $L$ and $Q$, and the number of eigenvectors should be determined by compromising between the dimension number of the resultant system and the approximation accuracy. Furthermore, the basis vectors obtained from the singular value decomposition are orthonormal, which gives us the following relations

$$
\left\langle\phi_{i}, \phi_{j}\right\rangle=\left\{\begin{array}{lll}
1 & \text { if } & i=j \\
0 & \text { if } & i \neq j
\end{array}\right.
$$

and the general Fourier coefficients in this case could be calculated as

$$
a_{k}=\left\langle U^{k}, \phi_{k}\right\rangle \text {. }
$$




\subsection{Galerkin Projection}

The idea of constructing a lower dimensional dynamic system from a given higher dimensional system is to replace the dynamics of the given system by a lower dimensional subspace of the origin state space [7,5, 12. Following the standard procedure, we substitute the approximation Eq.(4) into the given dynamic system Eq.(3), with the basis vectors extracted from the collection matrix $U$, and write it in the residual form

$$
r(x, t)=\frac{\partial \mathcal{U}(x, t)}{\partial t}-\mathcal{F}(\mathcal{U}(x, t))=\sum_{k=1}^{M} \frac{\partial a_{k}}{\partial t} \phi_{k}-\mathcal{F}\left(\sum_{k=1}^{M} a_{k} \phi_{k}\right) .
$$

Then, we use a Galerkin projection so that we approximate the system by a lower dimensional subspace approximation, and set the residual, induced by this approximation, to be orthogonal to all the basis functions

$$
\left(r, \phi_{k}\right)=\int_{\Omega} r(x) \phi(x) d x=0 .
$$

In the discrete case, we set the inner product between the residual vector and all the basis vectors to zero, so that for the given dynamic system, it is easy to get that

$$
\left\langle\sum_{k=1}^{M} \frac{\partial a_{k}}{\partial t} \phi_{k}, \phi_{j}\right\rangle=\left\langle\mathcal{F}\left(\sum_{k=1}^{M} a_{k} \phi_{k}\right), \phi_{j}\right\rangle .
$$

Since the $\phi_{j}$ produces a set of orthonormal basis vectors, the system can be re-cast into the following set of ODEs

$$
\frac{\partial a_{j}}{\partial t}=\left\langle\mathcal{F}\left(\sum_{k=1}^{M} a_{k} \phi_{k}\right), \phi_{j}\right\rangle, \quad k, j=1, \ldots, M .
$$

Since the basis vectors are extracted from the snapshots, all the boundary conditions are "embedded" into the basis vectors which help avoid a stiff system of equations. The resulting system of ODEs is integrated given the initial conditions projected into the orthonormal basis, and the loadings are applied.

\section{$4 \quad$ Numerical Results}

In what follows we demonstrate the technique described above on the empirical low dimensional model by simulating the dynamical behavior of a SMA rod. The dynamical behavior of the SMA rod is strongly nonlinear with coupling effects between the elastic and thermal fields, first order martensitic transformations,

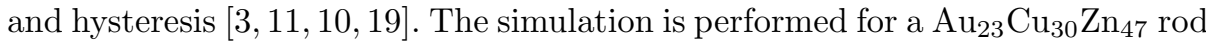
of length $L=1 \mathrm{~cm}$ based on Eq.(11). All the physical parameters for this specific material are taken here the same as in [11. We use the following procedure. 

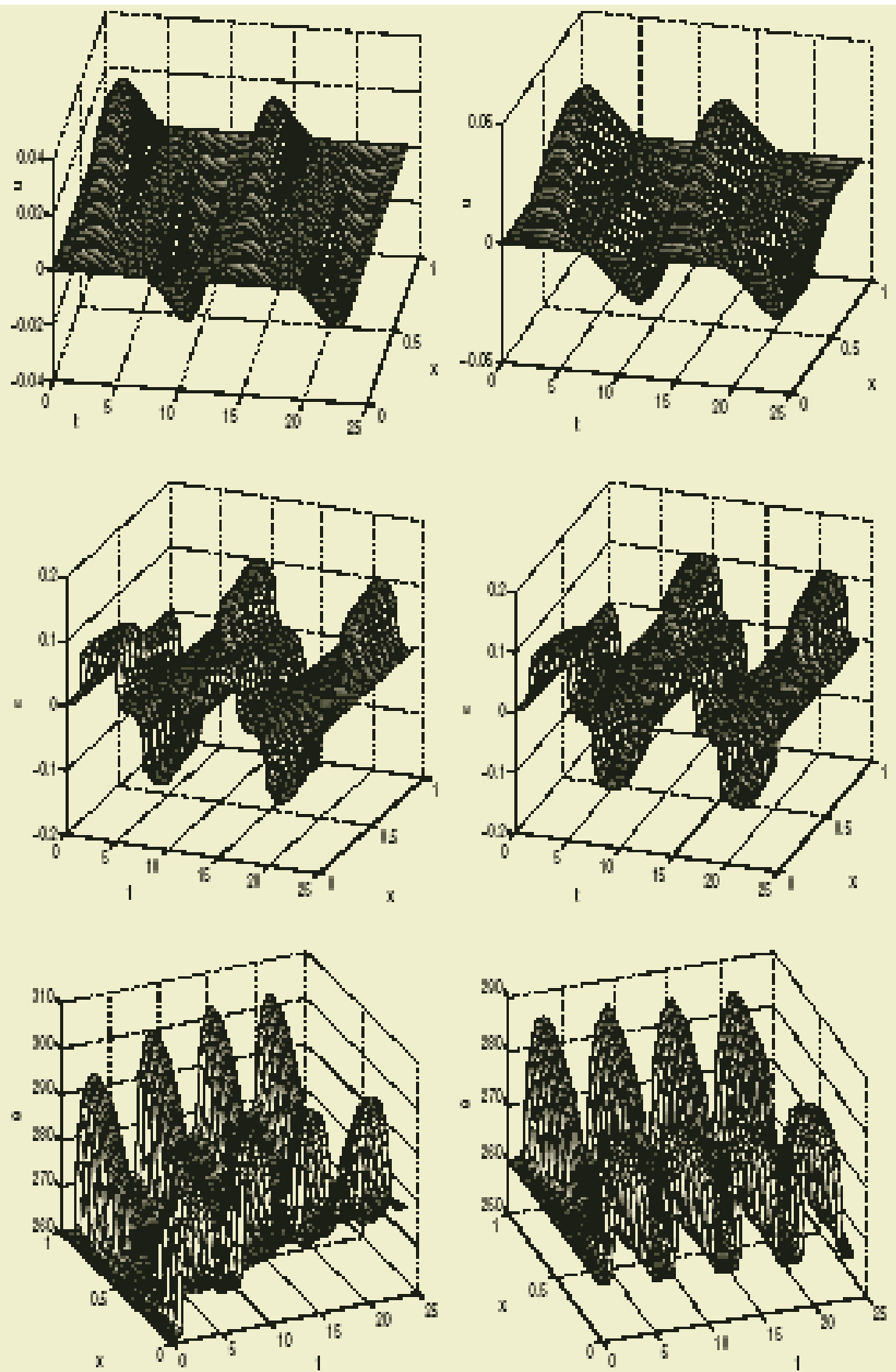

Fig. 1. Comparison of the nonlinear thermo-mechanical behavior of a SMArod: the full PDE model (left) and the empirical low dimensional model (right) 
First, we perform the numerical simulation using Eq.(1) with a representative mechanical load. As a result, the collection matrix will be constructed and the empirical orthonormal basis vectors can be extracted. Then, we simulate the dynamics using Eq.(14) with different mechanical loads. By comparing the numerical results from Eq.(14) with those from Eq.(11), we analyse the performance of the low dimensional model in reproducing the main features of the dynamics.

The initial conditions for all simulations are set the same. In particular, $\theta(x, 0)=260^{\circ} K$ and $\epsilon(x, 0)=v(x, t)=0$. Boundary conditions for Eq.(1) are taken as mechanically pinned-end and thermally insulated. The distributed mechanical loading for collecting snapshots is as follows (for one period)

$$
F=7000 \begin{cases}t / 3, & 0 \leq t \leq 3 \\ (6-t) / 3, & 3 \leq t \leq 9 \\ (t-12) / 3, & 9 \leq t \leq 12 .\end{cases}
$$

We follow here ideas reported in [11,10,19]. There are 18 nodes used for $\epsilon$ and $\theta$ discretization and 19 nodes used for $v$. Two periods of loadings are performed $(t \in[0,24])$ and totally 201 evenly distributed snapshots are sampled.

After the POD is applied, there are 9 basis vectors that are kept for $\epsilon, 9$ for $v$, and 8 basis vectors for $\theta$. By substituting the approximation

$$
u=\sum_{i=1}^{9} \epsilon_{i} \phi_{i}^{\epsilon}, \quad v=\sum_{i=1}^{9} v_{i} \phi_{i}^{v}, \quad \theta=\sum_{i=1}^{8} \theta_{i} \phi_{i}^{\theta}
$$

into Eq.(1), the system will be converted into a system of ODEs with dimension of 26 ( 9 for $\epsilon, 9$ for $v$, and 8 for $\theta$ ). Then, a standard ODE integrator (ode23 in Matlab) is applied to simulate the state evolution, without changing any physical parameters, except the mechanical loadings that is now $F=6000 \sin (\pi t / 6)^{3}$.

The numerical results obtained with the low dimensional model are presented in the right column of Fig.1. The displacements (displacement is calculated by integrating $\epsilon$ along $x$ after simulation), temperature and strain distributions in the entire rod are plotted as functions of time. For comparison purposes, the behavior of the SMA rod with exactly the same physical parameters and mechanical loading is also simulated using Eq.(1), and the numerical results for displacement, temperature and strain are presented in the left column of Fig.1, in a similar way. The validation of the numerical results obtained with Eq.(1) has been previously discussed in [19,11]. By analysing Fig. 1, we conclude that all the characteristic features of the material are captured by the constructed here lower dimensional model.

\section{References}

1. Atwell,J.A., Borggard,J.T., King,B.B.: Reduced Order Controller for Burgers' Equation With a Nonlinear Observer. Int.J.Appl.Math.Comput.Sci. 611 (2001) 1311-1330.

2. Beeler,S.C., Tran.H.T., Banks.H.T.: Feedback control methodologies for nonlinear systems, Journal of Optimization Theory and Application, 1107 (2000) 1-33 
3. Falk, F., Konopka, p.: Three-dimensional Landau theory describing the martensitic phase transformation of shape memory alloys. J.Phys.:Condens.Matter 2 (1990) 61-77.

4. Fattorini,H.O.: Infinite Dimensional Optimization and Control Theory. Cambridge University Press, (1999) Cambridge.

5. Holmes,P., Lumley,J.L., Berkooz,G.: Turbulence, Coherent Structures, Dynamical systems and Symmetry. Cambridge University Press, (1996) Cambridge.

6. Hu,M, Du,H.J., Ling,S.F., Zhou,Z.Y., Li,Y.: Motion Control of Electrostrictive Actuator. Mechatronics, 214 (2004) 153-161

7. Kerschen,G., Colinval,J.C.: Physical Interpretation of the Proper Orthogonal Modes Using the Singular Value Decomposition. Journal of Sound and Vibration. 5249 (2002) 849-865.

8. Liang,Y.C., Lee,H.P., Lim,S.P., Lin,W.Z., Lee,K.H., Wu,C.G.: Proper Orthogonal Decomposition And Its Applications-Part I:Theory. Journal of Sound and Vibration, 3252 (2002)527-544.

9. Lurie,K.A., Applied Optimal Control Theory of Distributed Systems. Plenum Press, (1993) New York.

10. Melnik, R., Roberts, A., Thomas, K.: Coupled thermomechanical dynamics of phase transitions in shape memory alloys and related hysteresis phenomena. Mechanics Research Communications 286 (2001) 637-651.

11. Melnik, R., Roberts, A., Thomas, K.: Phase transitions in shape memory alloys with hyperbolic heat conduction and differential algebraic models. Computational Mechanics 29 (1) (2002) 16-26.

12. Melnik, R.V.N. and Roberts, A.J., Modelling nonlinear dynamics of shape-memory alloys with approximate models of coupled thermoeleasticity. ZAMM: Zeitschrift fur Angewandte Mathematik Mechanik 83 (2) (2003) 93-104.

13. Meyer,M., Matthies,H.G.:Efficient Model Reduction in Nonlinear Dynamics Using the Karhunen-Loeve Expansion and Dual-Weighted Residual Methods. Computational Mechanics 31 (2003) 179-191

14. OR,A.C., Kelly,R.E.: Feedback Control of Weakly Nonlinear Rayleigh Bernard Marangoni Convection. J.Fluid.Mech., 440 (2001) p27-47.

15. Rowley,C.W, Colonius,T., Murray,R.M.: Model Reduction for Compressible Flows Using POD and Galerkin Projection. Physic D 189 (2004) 115-129.

16. Rowley,C.W., Marsden,J.E.: Reconstruction Equations and Karhunen-Loeve Expansions for Systems with Symmetry. Physica.D 142 (2000) 1-19.

17. Sood,D.K, Lawes,R.A., Varadan,V.V (Eds): Smart Structures and Devices, Proceedings of SPIE, December 2000, Melbourne, Australia.

18. Teman,R.: Infinite Dimensional Dynamical Systems in Mechanics and Physics. Springer-Verlag (1998) New York

19. Wang, L., Melnik, R.: Nonlinear coupled thermomechanical waves modelling shear type phase transformation in shape memory alloys. in Mathematical and Numerical Aspects of Wave Propagation, Eds.G.C.Cohen, et al,Springer,723-728 (2003). 